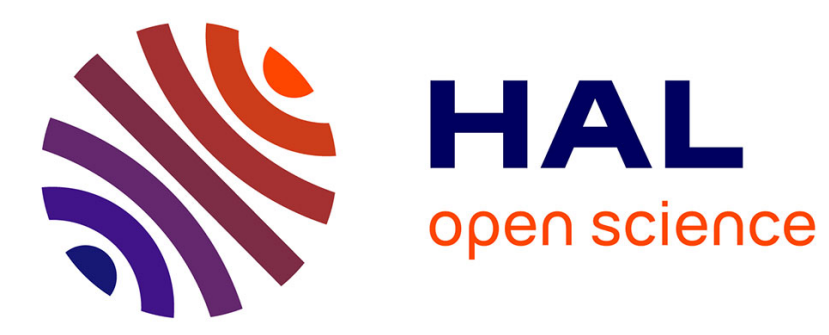

\title{
Limb versus Speech Motor Control: A Conceptual Review
}

Britta Grimme, Susanne Fuchs, Pascal Perrier, Gregor Schöner

\section{To cite this version:}

Britta Grimme, Susanne Fuchs, Pascal Perrier, Gregor Schöner. Limb versus Speech Motor Control: A Conceptual Review. Motor Control, 2011, 15 (1), pp.5-33. 10.1123/mcj.15.1.5 . hal-00565496

\section{HAL Id: hal-00565496 \\ https://hal.science/hal-00565496}

Submitted on 13 Feb 2011

HAL is a multi-disciplinary open access archive for the deposit and dissemination of scientific research documents, whether they are published or not. The documents may come from teaching and research institutions in France or abroad, or from public or private research centers.
L'archive ouverte pluridisciplinaire HAL, est destinée au dépôt et à la diffusion de documents scientifiques de niveau recherche, publiés ou non, émanant des établissements d'enseignement et de recherche français ou étrangers, des laboratoires publics ou privés. 


\title{
Limb versus Speech Motor Control: A Conceptual Review
}

\author{
Britta GRIMME ${ }^{1}$, Susanne FUCHS ${ }^{2}$, Pascal PERRIER $^{3}$, Gregor SCHÖNER $^{1}$ \\ ${ }^{1}$ Institut für Neuroinformatik, Ruhr-Universität Bochum, Germany \\ ${ }^{2}$ Center for General Linguistics (ZAS), Berlin, Germany \\ ${ }^{3}$ DPC//GIPSA lab, UMR CNRS 5216, Grenoble INP, France
}

Emails:

britta.grimme@ini.rub.de

fuchs@zas.gwz-berlin.de

Pascal.Perrier@gipsa-lab.grenoble-inp.fr

gregor.schoener@ini.rub.de

Motor Control, 15(1), 5-33

January 2011 


\begin{abstract}
This paper presents a comparative conceptual review of speech and limb motor control. Speech is essentially cognitive in nature and constrained by the rules of language, while limb movement is often oriented to physical objects. We discuss the issue of intrinsic vs. extrinsic variables underlying the representations of motor goals as well as whether motor goals specify terminal postures or entire trajectories. Timing and coordination is recognized as an area of strong interchange between the two domains. Although coordination among different motor acts within a sequence and co-articulation are central to speech motor control, they have received only limited attention in manipulatory movements. The biomechanics of speech production is characterized by the presence of soft tissue, a variable number of degrees of freedom, and the challenges of high rates of production, while limb movements deal more typically with inertial constraints from manipulated objects. This comparative review thus leads us to identify many strands of thinking that are shared across the two domains, but also points us to issues on which approaches in the two domains differ. We conclude that conceptual interchange between the fields of limb and speech motor control has been useful in the past and promises continued benefit.
\end{abstract}


Limb versus Speech Motor Control: A Conceptual Review

\author{
Introduction
}

Movement is an essential part of human experience. We move by walking, running, talking, and singing without thinking about the complex dynamical processes necessary to realize these motions. To reach for a cup on a table or to articulate a single word, we need to determine motor goals, identify movement parameters, initiate time, and coordinate the movement of different parts of the motor apparatus, and bring about the physical motion through the muscular system. As a semiotic task ruled by spoken language, speech appears to be quite different at first sight, and this difference is reflected in the existence of two separate research areas of speech vs. limb motor control. This paper presents a comparative review of scientific work in both domains and aims at identifying common strands of thinking as well as issues on which the two domains differ. Such a comparison may lead to insights into shared principles between the two forms of motor activity as well as to the identification of particularities of the two systems.

We organize our review in three parts. First, we analyze the problems of movement planning and control: Movement planning may formally be defined to comprise all processes of movement preparation that take place before a movement is initiated, while control entails the generation of time courses of relevant control variables which lead to the generation of planned motor acts. Movement planning and control are quite intimately linked. The time courses of control variables reflect motor goals, possible optimization principles, and predictable perturbations. Conversely, movement plans can be updated during the preparation or execution of a motor act. When sequences of motor acts are performed, as is typically the case in the production of speech, the processes of movement preparation and control overlap temporally and are intricately interwoven.

Next, we focus on two other closely associated aspects of movement generation: timing and coordination. Timing is critical to speech production, since it can carry relevant information for the communication process, and generally involves the 
coordination of different end-effectors and different movements. Most goal-directed arm movements are equally timed, even when such timing does not appear to be intrinsically required by the purpose of the task. Therefore, we discuss the general principles of timing and coordination that seem to underlie voluntary movement across many different tasks.

Finally, in a shorter section, we discuss the main issues in movement execution including control and biomechanical aspects of neuromusculoskeletal effector systems. Here, the articulatory apparatus displays a number of particularities in speech production that are worth further investigation.

In all cases, our emphasis is on comparing the conceptual and theoretical frameworks used in the two fields in addition to comparing the two types of motor acts themselves.

\section{Movement Planning and Control}

To illustrate the processes involved in planning and controlling a movement, we will take the reader through a scenario that highlights first a speech act and then a goal-directed hand movement. Think of a patron in a bar who asks for a glass of water and then drinks it. While catching the attention of the waiter, the customer selects and plans an appropriate sentence like "A glass of water, please!", and then begins to utter that phrase. This requires a sequence of well-coordinated orofacial movements. The word 'please', for instance, is made up of the four phonemes /pli:z/, each of which entails several articulatory subgoals that have to be reached within a very short time window of approximately $250 \mathrm{~ms}$. The labial stop /p/ requires a bilabial closure, in which the lips form an airtight seal, be followed by a rapid release, a sequence which generates first an acoustic silent period followed by the burst characteristic for stops. Simultaneously, the vocal folds must be abducted to suppress vibrations that would induce the perception of a $/ \mathrm{b} /$ instead of a $/ \mathrm{p} /$. During the oral closure for the $/ \mathrm{p} /$, the speaker anticipates the production of the subsequent $/ \mathrm{l} /$ that is realized with the tip of the tongue at the alveolars. Adduction of the glottis initiates vocal folds vibrations also required for $/ \mathrm{l} /$. The vowel /i:/ is then produced maintaining a relatively high tongue dorsum position which leads to the small front and large back vocal 
tract cavity that are necessary to generate the typical resonance frequencies of this vowel. Finally, the fricative $/ z /$ is produced with the tip of the tongue forming a tight constriction at the alveolars, which generates turbulent flow, while keeping a high jaw position and adducted vocal folds to maintain their vibrations. Moreover, the speaker lengthens the sequence /i:z/ to mark a phrase boundary.

After receiving the glass of water, the patron reaches for and grasps the glass and then brings it to his lips and drinks. The overall goal of this action may be decomposed into a number of subgoals. First the actor reaches for the glass and grasps it without overthrowing it and spilling all the water. During the transport phase of reaching movement as well as later while transporting the glass toward the mouth, any obstacles in the hand's path are avoided. Lifting the glass requires fine coordination of the normal forces of the grip to avoid slippage while accelerating the glass tangentially to the grasp surface. Finally, when approaching the lips with the glass, fine compliant control leads to contact and enables pouring the water into the mouth.

The first part of the afore described scene highlights typical features of speech articulatory movements: the existence of articulatory goals, which are lined up in complex serial order, the intricate coordination involved in achieving the articulatory goals, as well as co-articulation that occurs as different gestures overlap; all these gestures are aiming at transmitting cognitive information that will be processed by the waiter; his appropriate understanding of the information is a requirement for the second part of the scene to exist.

The second part of the scene emphasizes the importance of available environmental sensory information in order to enable the definition of several subgoals and the preparation of a goal-directed motor act composed from these subgoals. For each subgoal, movement parameters like direction, amplitude, and accuracy of the movement must be determined at least in part before the motor act is initiated. For instance, the initial movement direction of the hand reflects the direction toward the target. Larger and faster movements begin faster and an expectation to encounter mechanical resistance leads to stronger force generation from the very start of the movement. This section will begin with an analysis of motor goals. Evidence will be provided that motor goals in speech motor 
control differ from the motor goals of simple object-oriented movements such as grasping or pointing. We will also see, however, that many core questions of research in motor control are shared across the two systems. Of these, we will examine two in depth: (1) Are motor goals extrinsic or intrinsic? (2) Do motor goals specify only the final target position or do they constrain the entire movement trajectory?

\section{Extrinsic versus Intrinsic Motor Goals in Limb Movements}

Motor goals are extrinsic if the Central Nervous System (CNS) specifies the spatial trajectory or the target equilibrium position of the task's end-effector (i.e. tool point or terminal device for the corresponding effector system) in the outer visual space, such as a finger's end-point in a pointing task. Motor goals are intrinsic if defined in an effectorsystem-specific manner. Thus, intrinsic goals could be joint angles or forces generated by limb or arm muscles. Below we will criticize this conceptual distinction and try to dissolve it. The quest to identify the variables in terms of which motor goals are represented has, however, been a major theoretical theme in the field.

The extrinsic motor goals hypothesis was introduced by Bernstein (1935) who assumed that "there exist in the higher levels of the CNS projections of space and not projections of joints and muscles" (cited by Morasso, 1981, p. 224, similar views had been voiced by Hughlings-Jackson as early as 1899). In modern movement science, the debate about extrinsic vs. intrinsic motor goals began with studies which found that some key properties of the movement trajectories of the end-effector were invariant in the outer Cartesian space, across repetitions, conditions, and subjects: movement paths of the endeffector are gently curved and their tangential velocity profiles are bell-shaped with a single peak (Morasso, 1981; Abend et al., 1982). Soechting and Lacquaniti (1981), studied pointing arm movements in a vertical plane and interpreted extrinsic invariants as the consequence of the temporal coordination of the joint angles at the shoulder and the elbow (see Flanagan and Ostry (1990) for a similar conclusion for jaw movements). Uno et al. (1989) and Kawato et al. (1990) argued that optimization principles may add intrinsic to 
extrinsic constraints. They proposed that human movements are planned in terms of a number of via-points specified in the extrinsic Cartesian space of the end-effector. The realization of these extrinsic goals would then be achieved by optimizing intrinsic variables such as the change of the torque at each joint. Force-field studies probe this hypothesized combination of extrinsic constraints within intrinsic optimization. When Shadmehr and Mussa-Ivaldi (1994) asked participants to move the handle of a robotic manipulandum onto which an initially unknown force field was applied, participants learned to produce endeffector trajectories similar to the ones observed in the absence of a force-field. To achieve this invariant extrinsic motor goal, the participants had to generate quite different torque profiles at the intrinsic level. This amounted, according to the authors, to learning an internal model of the external force field.

Because extrinsic and intrinsic variables are necessarily in a one-to-one relationship in non-redundant ${ }^{\mathrm{i}}$ effector systems, those experiments — all based on such systems — are fundamentally inconclusive with respect to the relationship between these two frames of reference. Any extrinsic constraint can always be recast as an intrinsic constraint and vice versa. Most human effector systems are redundant for most typical human motor tasks, however, both at the kinematic level and at the level of muscles (Latash et al., 2007). In a redundant effector system, multiple possible intrinsic kinematic states are possible for any given extrinsic goal. Typically, the set of such task-equivalent joint or muscle configurations forms a continuous set, sometimes called the "uncontrolled" or self-motion manifold (Schöner, 1995). Scholz and Schöner (1999) have proposed that the structure of variance in the intrinsic space can reveal motor goals in the extrinsic space. Specifically, they provided a method to decompose variance at the joint level into joint configurations that leave extrinsic variables invariant and joint configurations that induce variance of extrinsic variables. Evidence for extrinsic motor goals is obtained if variance that affects the extrinsic variables is suppressed compared to variance that does not affect the extrinsic variables. This signature was found in a great variety of tasks and effector systems that ranged from the sit-to-stand transition (Scholz \& Schöner, 1999), upright stance (Hsu et al., 2007), pointing in 3D (Tseng et al., 2003) to shooting (Scholz et al., 2000). How multiple 
muscles generate a particular level of torque and stiffness at any individual joint can similarly be analyzed using the concepts of the uncontrolled manifold (Latash et al., 2007; Krishnamoorthy et al., 2005, for an early proposal see Laboissière et al., 1996). A theoretical model showed how the coupling between extrinsic motor goals and intrinsic joint-level variables may give rise to the structure of variance that the uncontrolled manifold describes while at the same time providing for classical invariance effects (Martin et al., 2009). This theory suggests that motor systems are structured by constraints at the level of extrinsic task variables, which are realized, however, in the form of coupling structures at the level of the intrinsic effector variables.

\section{Extrinsic versus Intrinsic Motor Goals in Speech Production}

During speech production, the movements of the articulators create temporal sequences of sounds. At the intrinsic level, activations of the tongue, jaw, lips, vocal folds and velum muscles, i.e. movements of the vocal tract articulators, and the resulting vocal tract shapes correspond to the produced patterns of speech. At the extrinsic level, acoustic signals similarly correspond to the produced patterns of speech. In this respect, the question of intrinsic vs. extrinsic motor goals may also be posed in speech production. However, the semiotic nature of speech complicates the picture. Indeed, speech gestures and speech sounds are produced to carry abstract linguistic information that is transmitted from the speaker's brain to the listener's brain. Hence, contrary to object-oriented reaching or grasping movements, the objectives of speech production are defined relative to linguistic information and communicative intentions of the speaker, but not relative to the directly accessible physical world. For most communicative intentions, the physical characteristics of speech production have no meaning by themselves but make sense only in relation to the listener's perception. As a result, the physical correlates of speech production can be highly variable without invalidating the perception of speech. Thus, issues about the nature of motor goals in speech production have been widely discussed in the context of the production-perception interaction, taking into account the structure of the language shared by speakers and listeners. 
Four major theories have provided the frameworks for a large amount of experimental and modeling work and have been at the core of numerous controversial debates (Perkell \& Klatt, 1986). The Acoustic Invariance Theory (Stevens \& Blumstein, 1978; Blumstein \& Stevens, 1979), the Quantal Theory of Speech (Stevens, 1972, 1989), and the Adaptive Variability Theory (Lindblom, 1988, 1990) basically defend the idea that the motor goals are in the auditory perception domain. Thus, in the language of motor control these theories favor extrinsic motor goals. In contrast, Stetson (1928) claimed that "Speech is rather a set of movements made audible than a set of sounds produced by movements." (Stetson, 1928, p. 29). In line with Stetson, the Motor Theory (Liberman et al., 1967; Liberman \& Mattingly, 1985), and the Direct Realist Theory (Fowler, 1986, 1991) defend the idea that the goals of speech production lie in the articulatory domain. In the language of motor control, these goals are intrinsic in nature.

Although originally dealing with speech perception, the four theories have strongly influenced experimental and theoretical research in speech motor control, as speech production and perception are intimately linked. Moreover, these theories are strongly connected to some influential phonological theories. The Acoustic Invariance Theory and the Quantal Theory have been developed in relation with the Features theory elaborated by Chomsky \& Halle (1968). The Motor Theory and the Direct Realist Theory are linked with Articulatory Phonology (Browman \& Goldstein, 1986).

Methodologically, the approach to the extrinsic vs. intrinsic issue has been similar in studies of limb motor control and studies of speech production. In speech, several studies have looked for invariants, either in the extrinsic acoustic domain (Stevens \& Blumstein, 1978; Strange et al., 1983; Sussman et al., 1998), or in the intrinsic domain (Fujimura, 1986; Boë et al., 1992; Tremblay et al., 2003; Baer et al., 1988; Maeda \& Honda, 1994). Analogously to what force-fields do for limb movement, perturbation paradigms have been elaborated to break down the usual associations between extrinsic (acoustics) and intrinsic (articulation) variables. These perturbations prevented the speakers from achieving their natural articulatory gestures (Gay et al., 1981; Savariaux et al., 1995) by modifying the vocal tract geometry (McFarland et al., 1996), distorting the 
orosensory (Borden et al, 1973; Kelso \& Tuller, 1983) or the auditory feedback (Houde \& Jordan, 1998; Purcell \& Munhall, 2006), as well as by altering the relation between the visually perceived articulation and the auditory perceived acoustic signal (McGurk \& MacDonald, 1976).

An analogy at the conceptual level is the notion of redundancy. The cognitive function of speech in a communicative act permits variability of a certain kind. Words can be partially identified on the basis of the temporally and spectrally changing physical signal, the semantic context, the frequency of their use, the pragmatic expectations of the listener, and/or the complexity and form of the phonological or morphological structure. Thus, the production of speech involves a highly redundant physical system that projects onto a cognitive space.

Similarly to what emerged for limb motor control research, almost 50 years of discussion and controversy seem to lead to the conclusion that under normal conditions the motor goals of speech production are both auditory (i.e. extrinsic) and articulatory (i.e. intrinsic). This view is defended among others by Guenther et al. (1998) in his DIVA model or by Schwartz et al. (2010) in the Perception-for-Action-Control Theory (PACT). They consider speech gestures not merely as articulatory units, but as patterns of motor coordination shaped by motor-to-auditory nonlinearities. Perrier (2005) suggested that under normal conditions the multi-modality of the speech goals enables the motor system to adapt control strategies to context. Based on an analysis of various speech perturbation experiments, Perrier concluded that the articulatory and auditory modalities do not have equivalent status, the primary goal of speech lying in the auditory domain.

This suggests that a speech production analogue of the "uncontrolled manifold hypothesis" might be useful (Schöner, Martin, Reimann, Scholz, 2008, Saltzman, Kubo, Tsao, 2006). Such a hypothesis would suggest that task-level goals structure the coupling among the components of the articulatory apparatus such that task-equivalent configurations may be generated depending on context, perturbations, and the demands of additional task dimensions (Martin et al., 2009). It is then the task level that determines which patterns of variability are compatible with the action goals at any moment in time. 
Developing such a hypothesis and the associated tools of analysis may help understanding and predicting the amount of variability that is compatible with the relevant communicative act.

\section{Endpoint Control or Trajectory Control in Limb Movements}

A second, related theoretical issue of motor planning and control is the question if motor goals specify merely the target position or configuration of the effector systems or whether motor goals represent an entire movement trajectory. In the first case, the time course of movement would emerge from neuro-muscular processes that are independent of motor goals. In the second case, the time course of movement results from complex neural processes that are shaped by motor goals.

A direct test of the notion of a planned trajectory was proposed by Polit and Bizzi (1979) who studied the effect of perturbations applied during arm movements of large amplitude in the absence of visual feedback in monkeys. In normal and in deafferented monkeys the perturbations did not prevent the animals from attaining the final target position, consistent with specification of the final position only. However, Bizzi et al. (1984) found in a refined experiment that following a brief phasic perturbation monkeys moved their deafferented arm back toward the unperturbed trajectory before reaching the final target position. This supported the notion of a virtual trajectory, a continuous sequence that is planned either in the intrinsic or in the extrinsic domain.

To explain how the virtual trajectory is elaborated, two main trends exist in motor control research. The first one proposes that this trajectory results directly and simply from the properties of the initial and final positions of the movement, without any specific requirements for the trajectory itself. The second one considers that the virtual trajectory results from complex processes involving internal representations of the motor system and planning, in order to match some requirements during the movement from the initial to the final position. The debate is exemplified by a controversy that came up in the late nineties between Gomi and Kawato (1996) and Gribble et al. (1998). Gomi and Kawato (1996) measured the trajectories and velocity profiles of two-joint (shoulder and elbow) planar arm 
movements and estimated the temporal evolution of the joints' stiffness during the movement. Using a simple linear second-order model, they estimated the virtual trajectories of the joint-muscle equilibrium points. Because these trajectories were complex, the authors concluded that they could not be derived from a peripheral mechanism that is only controlled by the desired target configuration, requiring instead complex computations based on internal models of the motor system. In a reaction to this paper, Gribble et al. (1998) considered the same kind of data. They interpreted in terms of the Equilibrium Point Hypothesis (Feldman, 1986), in which the length of virtual muscle equilibrium varies at piecewise constant rates during the movement. These are determined entirely by the target configuration. Their muscle model had considerably more physiological detail and included a strongly non-linear torque-length relationship which was sufficient to account for the seemingly complex kinematic and stiffness data.

The $2 / 3$ power law, that links movement path curvature and speed (Viviani \& Stucchi, 1992), has been interpreted as a signature of optimal planning of the movement trajectory and in that respect as evidence for control of the entire trajectory rather than only of the movement end point (Viviani \& Flash, 1995). This interpretation of the $2 / 3$ power law has, however, been called into question by Schaal \& Sternad (2001). While acknowledging that jerk minimization generates smooth trajectories consistent with the $2 / 3$ power law, these authors showed that such trajectories may also arise from the spring-like properties of the motor system. Convergent evidence comes from Gribble and Ostry (1996) and Perrier and Fuchs (2008) who were able to simulate the speed-curvature relations for planar arm movements and for tongue movements using realistic biomechanical models without planning at the trajectory level.

This exemplifies broader issues of the status of a large amount of theoretical work that invokes optimality principles such as minimum jerk (Hogan, 1984; Flash \& Hogan, 1985), minimum work (Soechting et al., 1995), minimum-torque (Uno et al., 1989), and minimum final state variance (Harris \& Wolpert, 1998). In all cases, the underlying assumption is that the trajectory as a whole is the object of optimization and with it planning, either explicitly or through the underlying mechanism of torque generation. In 
stochastic optimal control, such trajectory planning is updated at any time during movement execution, a notion in which planning and feedback control are intertwined (Todorov \& Jordan, 2002). However, Hermens and Gielen (2004) found out that none of these optimal control models could account well for experimental data collected from humans in a reaching task in 3D space.

To date, the debate about end-point vs. trajectory based motor planning has not been definitely settled and continues to be an important topic in motor control research that is linked to other major conceptual issues such as the existence and the complexity of internal models, and the role of short delay feedback in movement control ${ }^{\mathrm{ii}}$. There is also the possibility, that the question has no general answer. The extent to which movement trajectories are planned as a whole may depend on the extent to which the precise timing of the trajectory matters (see also the next section on timing).

\section{Endpoint Control or Trajectory Control in Speech Production}

The classical description of phonological inputs as sequences of discrete phonemes suggests that speech production is organized in terms of movement targets, each associated with a phoneme. The question whether speech motor control is fully determined by the characteristics of the targets or whether additional constraints apply to the transitions between targets is thus linked to phonological theories. Target based speech motor control is consistent with the concept of the phoneme and is much debated in phonology (Ohala, 1983; Ladefoged, 2000; Cutler et al., in press). If the trajectories between targets are controlled, then this may be consistent with theories advocating larger units which attribute an important role to transitions rather than to steady states per se.

Theories in favor of target control are numerous. They differ with respect to the domain in which the target is realized. For instance, Fant (1960) or Stevens (1972) proposed that vowels are characterized by steady state spectral characteristics in the acoustic domain. Similarly, Blumstein and Stevens (1979) suggested acoustic targets (spectral characteristics of the burst) of stop consonants. In the articulatory domain, the 
location and the size of the constriction in the vocal tract are often assumed to characterize the vowel or consonant target (Browman \& Goldstein, 1986; Boë et al., 1992; Guenther, 1995). Payan and Perrier (1997) and Perrier et al. (2003) were able to synthesize complex velocity profiles and articulatory paths for selected phonemes using a biomechanical tongue model. In this model movements are generated based on shifts of the motor commands with constant rate between successive phoneme-specific target values.

Other studies provided evidence against the target hypothesis. Delattre et al. (1955) noticed that the spectral transition from a consonant to the following vowel plays a role in the perceptual identification of the consonant. Strange et al. (1983) studied the identification of vowels in Consonant-Vowel-Consonant sequences when the initial, the central, or the final part of the vowel was removed. She observed that when the center was removed, the error rate remained close to the error rate observed under normal conditions. In contrast, the error rate increased significantly when the initial or final part was removed, which belongs to the transition phase. These findings thus speak for a major role of the trajectory/transition in the identification sounds in general. More recently, Cai et al. (2008) applied an online perturbation paradigm to the first two formants of the /iau/ triphthong of Mandarin Chinese. They observed that speakers compensate for the perturbation to generate a trajectory in the first and second formant plane that is closer to the normal condition than it would be without compensation.

As for limb motor control, the question of whether movement targets or movement trajectories are the relevant motor goals continues to be a debated one in the speech production literature. In speech perception, it is now clear that steady states as well as transitions between steady states provide information that subserve the identification of phonemes. Transitions play a critical role in conveying contextual information. However, it is still unclear whether these transitions emerge from the intrinsic properties of the speech production system without explicit planning and then become key properties for the identification of phonological categories or whether they are intentionally produced and controlled to enable effective perception.

Although the fact that these debates continue is shared across the two domains, the terms in 
which these debates are framed differ substantially. In limb motor control, the debate centers on the contribution of the intrinsic dynamic properties of the motor system to trajectory formation, the existence and complexity of internal models, and the search for optimality principles governing trajectory formation. In speech motor control, the debate centers on the nature of the phonological units (phonemes, gestures or syllables) and the role of dynamics in speech production and perception. That said, it should also be noted that the theoretical and methodological approaches of the field of limb motor control have impacted on the study of speech motor control (see, among many others, Saltzman \& Munhall, 1989; Houde \& Jordan, 1998; Tremblay et al, 2008, Perrier \& Ma, 2008).

\section{Timing and Coordination}

Up to this point we have looked only into a small portion of what happens during real object-oriented motion and natural speech generation. We have focused on planning and controlling a single movement, while the timing and coordination of multiple motor acts are also critical aspects of motor control. In the glass-of-water scene, the reaching movement of the hand to the glass of water unfolds at the same time as the hand is prepared for the grasp. Also the tilting movement of the hand to dip the glass before drinking is concurrent with the transport back as well as with the anticipatory opening of the mouth. Obviously, the coordinated interplay of all sub-movements over time is essential to reach the glass without throwing it over during grasping or spilling water during drinking.

Similarly, the timing of articulatory movements and the coordination of the ensemble of articulators are critical to successful speech production. The fast and variable speed of successive movements sets speech apart. In one breath most people are able to realize a sequence of 20 syllables or 40 phoneme targets, quite an extraordinary rapid fire sequence of action compared to the much lower rate at which object-oriented motor behaviors are generated. The high rate of articulatory motion is helped by the fact that most articulators, except the jaw, consist of soft tissue. The speed of speech articulatory movements has two important implications for speech motor control: First, the high rate generically creates considerable temporal overlap of the single movements associated with the production of 
adjacent phonemes. Successful speech generation requires that this overlap is properly managed, a phenomenon called coarticulation. Second, the high rate of articulatory movements makes online cortical feedback impossible! Moreover, the duration of speech gestures may convey perceptual meaning that is coded in a language (Isei-Jaakkola, 2004). Temporal transformations like stretching or shrinking of articulatory movements often signal boundaries between words, larger phrases, or sentences.

\section{Absolute and Relative Timing and its Implementation by Neuronal Limit Cycle Oscillators}

Timing is the control of the time course of an action. Two aspects of timing must be distinguished (see Schöner, 2002, for an introduction): Relative timing refers to the temporal coordination of multiple movements or effectors. Here, changes to the time course of one component affect the time course of other components. In this way, coordination establishes and maintains stable and reproducible temporal relationships between different effectors or between different movements of the same effector. Relative timing is typically assessed through the variability and stability of relative phase, or through the recovery of an invariant mean value of relative phase following a perturbation (Schöner \& Kelso, 1988). Absolute timing refers to how the duration of a movement is controlled to generate a rhythmic pattern (locomotion, dancing, chewing, etc.), or to align a movement with events in the outer world. Catching a ball is an example of a temporally discrete but timed motor act: the duration and point of initiation of the catching movement is determined by the perceived time to contact. If that time changes - e.g. due to wind that either holds up or accelerates the ball — the duration of the catching movement is adapted. Thus, absolute timing refers to the reproducibility and stability of the duration of a motor act and is typically assessed in terms of the variability of markers of duration (Ivry, 1996).

Most human movement involves both types of timing. This is true for speech production: Relative timing is critical to speech production in the form of the coordination of the many components of the articulatory apparatus such as the jaw, the tongue, the lips, and the larynx. Absolute timing plays a role when the duration of articulatory events is important for the communicative function. The importance of duration varies across 
different languages. In Japanese, we find relatively stable units of duration. In contrast, languages like English employ combinations of stressed and unstressed syllables, which differ in their durations, stressed syllables being typically longer in duration than unstressed syllables.

There has been much work on the neural mechanisms that may underlie both forms of timing. One concept is that of a neural clock which associates neuronal activation variables with time (Ivry \& Spencer, 2004). To become functional as a timing device, such a neural clock must be started and reset once the motor act has terminated. This makes such neural clocks special cases of the larger class of neural oscillators (Schöner, 2002). Stable oscillation is generic in coupled neuronal networks. An exemplary mathematical analysis by Amari (1977) makes intuitive, why that is the case. The mechanism entails an excitatory and an inhibitory neuronal population. The excitatory population couples both back onto itself (self-excitation) and onto the inhibitory population, which in turn projects inhibitorily onto the excitatory population. The analysis confirms the intuitive notion that such an arrangement will lead to a sequence in which an increase of excitatory activation is followed by an increase of inhibitory activation. The rising inhibition suppresses excitatory activation. When excitatory activation has fallen enough, it no longer drives inhibition so that inhibitory activation begins to fall. That releases excitatory activation from inhibition and the cycle can resume from the beginning. Similar circuits generating neuronal oscillation are common in the nervous systems.

Mathematically speaking, such stable neuronal oscillators form stable limit cycles which account for basic features of absolute timing and coordination (Schöner, 2002). In particular, the increase of timing variability with increasing mean duration (Wing \& Kristofferson, 1973), that is also observed in the perception of durations (Ivry \& Hazeltine, 1995), emerges naturally out of an oscillator account (Schöner, 2002). Such circuits also help understanding how timing is perceived (Ivry, 1996; Large \& Palmer, 2002) and how timed actions may be linked to perceived external signals (Schöner, 1994). Franz et al. (1992) provided evidence that timing processes involved in limb movements and those involved in speech movements overlap. They found that within-subject variability of cycle 
durations of repeated utterances, of non-speech jaw movements, of finger and of forearm tapping were all significantly correlated: Individuals with precise timing in arm or finger movement also tended to have precise timing in speech production.

\section{Coordination in Limb Movements}

Although coordination is often defined as the stabilization of both spatial and temporal relationships among the movements of different effectors or among movement components, stable relative timing is the most common signature of coordination. Relative timing can be assessed through the variance of measures of relative timing or by studying the recovery of a timing relationship after a phasic perturbation (Schöner \& Kelso, 1988; Turvey, 1990). The theoretical framework of neuronal oscillation lends itself to an account of coordination because the coupling among different neuronal oscillators generically leads to stable patterns of relative timing (Aronson et al., 1987). In fact, coupling two neuronal oscillators at the excitatory level leads to synchronization of the oscillators. If the oscillators and the motor systems which they drive are similar, the emergent pattern of relative timing is in an "in-phase" form of coordination in which homologous limbs go through identical kinematic states at the same time. If each inhibitory layer is coupled to the excitatory layer of the other oscillator, coordination manifests itself at "anti-phase" pattern synchronization, in which homologous components alternate their kinematic states (Schöner, 2002). These two patterns of coordination are generically found in rhythmic limb movement (Kelso, 1984). Without practice, other, intermediate phase relationships are more variable and biased toward these two basic patterns (Yamanishi et al., 1980).

That the stability of patterns of relative timing is not only a sufficient, but also a necessary condition for coordination is underlined by the observation that a loss of coordination is accompanied by a loss of stability (Schöner \& Kelso, 1988a). Kelso (1984) observed abrupt transitions from the anti-phase to the in-phase pattern of coordination when the frequency of rhythmic bimanual movement was increased. Later work confirmed the theoretical prediction (Schöner et al., 1986) that this transition should be accompanied by signatures of a loss of stability, that is, an increase of the variance of relative phase in 
the anti-phase pattern just prior to the transition (Kelso, Scholz, \& Schöner, 1986) and an increase in the time needed to recover the anti-phase pattern following a perturbation (Kelso et al., 1988).

May the theoretical account of coupled oscillators be interpreted mechanistically, for instance by postulating that coupling among spinal central pattern generators is responsible for the observed patterns of coordination and their instabilities (Grossberg et al., 1997)? Mechsner et al. (2001) have provided evidence, that the neuronal dynamics resides at a more abstract level that is closely linked to the perceptual representations of the movement patterns. In their experiments, participants performed coordinated movements of the index fingers of their two hands. The patterns were anatomically either in-phase (homologous muscles co-contracting) or anti-phase (homologous muscles alternating). In addition, the two hands were either held in symmetrical spatial postures (both hands facing up or both facing down) or in asymmetrical spatial postures (one hand facing up, the other facing down). As a result, the anatomical in-phase pattern was either a spatially symmetric pattern (the two index fingers moving toward each other) or a spatially asymmetric pattern (the two index fingers moving parallel to each other). The question was: is it always the anatomical anti-phase pattern that loses stability at higher frequencies? Or is it the spatial symmetry of the pattern that decides which pattern becomes unstable? Mechnser and colleagues found that the spatially asymmetric pattern, in which the index fingers move in parallel in space, is the one that becomes unstable, irrespective of whether that pattern is performed by homologous muscles alternating or by homologous muscles synchronizing! They concluded that coordination arises at the level of the spatial representation of planned and perceived movement (the ideo-motor principle, in their terms), not at the level of coupling between anatomical units.

Coupling and synchronization affect absolute timing. In a bimanual finger-tapping task, Helmuth and Ivry (1996) discovered that the variability of the cycle time for each effector was reduced compared to single-handed tapping (see also review by Ivry \& Richardson, 2002). The theory of coupled limit cycle oscillators explains this effect: Synchronization with a coupled oscillator is an additional source of stabilization 
(Schöner, 2002).

Although relative timing is often studied in rhythmic movements, stable temporal relationships occur naturally also during discrete motor acts. Kelso et al. (1979) contrasted mono-manual with bi-manual pointing movements. Using Fitts' law (1954) they arranged for one hand to perform a fast movement mono-manually (small amplitude, low precision requirement) and for the other hand to perform a slow movement mono-manually (large amplitude, high precision requirement). When the two disparate movements were initiated simultaneously with the two hands, their speed, velocity and acceleration patterns were almost perfectly synchronous. Similarly coordinated are the two components of an ordinary prehension movement, the reaching (or transport) component that moves the hand from its initial position to the spatial location of an object, and the grasping (or manipulation) component. Jeannerod (1984) found that the temporal relationship between these two components is highly stable, the fast initial phase of the transport component being synchronized with an extension movement of the fingers. The onset of finger closure is highly correlated with the beginning of the low velocity phase of the transport component which invariantly begins around 75\% of movement time. Paulignan et al. (1991) probed the stability of this pattern of coordination by inducing sudden and unexpected changes in the spatial locations of the reaching target. Corrective reaction was observed not only in the wrist's transport trajectory, but also in the kinematic pattern of hand shaping. The opening of the grip was interrupted and then resumed at the appropriate timing consistent with the approach movement. Analogous indices of coordination can be observed in considerably more complex movements (see Ivry et al., 2004, for a detailed review).

Theoretical modeling (Schöner, 1989) has demonstrated that the same conceptual framework, the coupling of stable limit cycles, can be used to account for the coordination of discrete movement. Temporally discrete motor acts require, of course, additional processes such as the initiation and termination of the movement, modeled through a second level of neuronal dynamics that drives the system through appropriate instabilities (Schöner, 1989). Thus, the shared mechanism of temporal coordination across discrete as well as rhythmic movement is not in conflict with neuronal (Schaal et al., 2004) and 
behavioral (Hogan \& Sternad, 2007) evidence for differences across these two forms of temporal organization.

\section{Interarticulatory Coordination in Speech}

Speech is fundamentally rhythmic in nature, if not in the sense of strict periodicity (Cummins \& Port, 1998). The temporal complexity of speech production makes the question of coordination all the more challenging and interesting. An emerging consensus in the research community postulates that during the production of each sound, different articulators are coupled over a brief time interval in a task-dependent manner. Compelling and direct evidence for the existence of stable, functional links among articulators during speech comes from perturbation studies. Kelso et al. (1984) and Munhall and Kelso (1985) developed an experimental design in which an unexpected mechanical load was applied to the participant's lower lip, preventing it to achieve the normal bilabial closure for the realization of $\mathrm{a} / \mathrm{p} /$ or $/ \mathrm{b} /$. Monitoring the movements of both upper and lower lip, these authors found a remote compensatory reaction in the upper lip, which-within about $40 \mathrm{~ms}$ after the onset of the perturbation - began to drop lower than on unperturbed trials to achieve the bilabial closure. Critically, this remote compensatory reaction was absent when bilabial closure was unnecessary (e.g., to utter/z/). The perturbations affected the timing of the subsequent sequence. When the onset of the perturbation occurred during the $/ \mathrm{p} /$, the subsequent vowel was shortened. When the offset of the perturbation occurred during the $/ \mathrm{p} /$, the subsequent vowel was lengthened. In a follow-up investigation (Saltzman et al., 1992; Munhall et al., 1994), unexpected perturbations were applied at three different points in time: just before the preceding vowel offset, early during closure, and late in the oral closure, that is, close to the release. In all cases, a longer acoustic Voice Onset Time (VOT) and shorter closure duration were found in the perturbed condition. In these studies, the researchers moved beyond the earlier work by also observing glottal abduction and adduction gestures by means of a transillumination technique. Laryngeal responses to the perturbation occurred mainly when the perturbation was applied just before the preceding vowel offset. The onset of glottal abduction was delayed causing a longer vowel duration, a 
delay that may be interpreted as an effort to preserve laryngeal-oral timing. This is consistent with the literature according to which the oral closing gesture and the glottal abduction gesture are closely timed in the production of voiceless stops (see Fuchs, 2005 for an overview). However, similar results were not found during the release phase or toward the end of /p/'s production. Thus, while the onset of laryngeal abduction and oral closure seem to be tightly coupled, this coupling seems less strong throughout the rest of the trajectory.

Differences in inter-articulatory coordination and timing between opening and closing gestures were reported by Gracco (1988) who looked at the coordination between the lips and the jaw in the first /p/ of the pseudo-word "sapapple". He found that the closing gestures of the two articulators were tightly coupled. In addition, Gracco examined the underlying EMG activities of the lips. While the timing of activation onsets, peak EMG amplitudes, and velocities for oral closing movements covaried, the oral opening turned out to be much more variable. This could be interpreted as consistent with the task requirements. The closing gesture in this example is the transition from the preceding vowel to the $/ \mathrm{p} /$ while the opening gesture is the transition from the $/ \mathrm{p} /$ to the following vowel. The closing gesture requires a tight coupling of the lips and the jaw but the opening gesture does not.

Indirect evidence for inter-articulatory coordination comes from studies of the labialcoronal effect and verbal transformation effect, in which slight changes of articulatory movements induce substantial shifts in the inter-articulatory coordination of successive segments and their perception. The labial-coronal effect goes back to MacNeilage and Davis (2000) who investigated speech acquisition and explained it in terms of the preferred co-occurrences between certain consonant and vowel places of articulations in human languages. According to MacNeilage and Davis, bilabials would be simpler to produce than coronals, since the first require only a cyclical jaw motion as in babbling and mastication, whereas the latter need an active motion of the tongue which is independent of jaw motion. They also proposed that the simpler consonant would be used to initiate a word. RochetCapellan and Schwartz (2007) suggested that the labial-coronal effect may also be 
explained in terms of an anticipation of the coronal consonant during the labial. To investigate inter-articulatory coordination during the labial-coronal effect these authors used a speeding up paradigm and found it to induce a shift from two jaw cycles per bisyllabic word to one jaw cycle. This shift modifies the interarticulatory coordination between the jaw and the constrictors: When the speaking rate increases in a sequence with a coronal consonant first and a labial second (CL), the change in jaw cycles may induce the reverse pattern with the bilabial consonant first (LC). In this sense, the LC sequence is more stable than the CL sequence. Sato et al. (2006) found that also in perception LCsequences are more stable (transform less) and more attractive (attract more transformations) compared to CL-sequences. Similar perceptual changes have been frequently found and are referred to as the verbal transformation effect. Repeating continuously the English word "life", for instance, can induce a shift from the perception of "life" to the perception of "fly" (for an overview, see Sato et al., 2006).

This brief review has illustrated that the study of the coordination among effectors is probably the area in which the closest theoretical and methodological synergy exists between approaches to limb and to speech articulatory movements.

\section{Intergestural Coordination in Speech: Coarticulation and Syllable Structure}

In speech production, the coordination between different segments that follow each other in time is a major theme. The traditional idea (Chomsky \& Halle, 1968) that language is generated from a limited number of invariant phonological units has not been confirmed empirically for a comprehensive set of sounds. At the level of the electromyographic, articulatory, or acoustic signals that accompany speech it is close to impossible to find evidence for such invariance (see Perkell \& Klatt, 1986, for a broad discussion). Thus, speech is highly variable. One potential origin of such variance is that in a sequence of units, neighbors may modify each others' physical characteristics. This phenomenon is called coarticulation and is one of the major topics in speech communication research that lies at the interface between phonetics and phonology. A central question is whether coarticulation originates from centrally controlled processes or whether coarticulation is 
merely a consequence of the mechanical properties of speech and is thus a relatively peripheral phenomenon. Another dimension of the literature on coarticulation is the distinction between the influence of a unit on its predecessor (regressive or anticipatory coarticulation) and the influence of a unit on its successor (progressive or carry-over coarticulation). It has been often proposed that anticipatory coarticulation may reflect a centrally planned process, while carry-over coarticulation could be due to the physical properties of the speech apparatus. Given the extensive research on coarticulation, we can only provide a cursory survey to illustrate the different streams in the field.

Henke (1966) studied anticipatory coarticulation. He supposed that phonemes are the minimal phonological units, characterized by a certain number of spectral or articulatory features. Features characterizing a phoneme, pn, would be propagated backwards to the preceding phonemes $\mathrm{pm}(\mathrm{m}<\mathrm{n})$ as long as they are compatible with the features of those phonemes pm. This look-ahead model is thus purely based on the linguistic feature description of the speech task.

In contrast, the co-production model elaborated at Haskins Laboratories posits that coarticulation is a consequence of physical interactions between temporally overlapping gestures (Fowler, 1977, 1980; Kelso, Saltzman, \& Tuller, 1986; Saltzman \& Munhall, 1989). In this model, speech units are specified as target gestures which are attractors of a dynamical control system, modeled as a linear mass-spring system. The gestures emerge from the transient relaxation of the articulatory system toward the attractor. The duration of these gestures thus depends on the strength of the attractor. Coarticulation takes place when the transient state of the articulatory system still reflects the previous gesture as the new attractor for the following gesture is set.

The co-production model has been extended to account for the coupling of gestures in onset and coda position and the way these positions affect the coordination of various articulatory gestures. In the planning oscillator framework an entrained ensemble of oscillators (one per gesture) collectively represent a multidimensional 'clock' for the utterance (Nam \& Saltzman, 2003; see also experimental data by Marin \& Pouplier, 2010). The model postulates that in the syllable onset position, coordination between all 
consonants (Ci) and the vowel (V) involve in-phase target states of the oscillators. If the onset consists of a consonant cluster (CC), then the two consonants are coupled in an antiphase pattern. These different forms of coupling lead to competition that gives rise to the ccenter effect (Browman \& Goldstein, 2000) in which any additional consonant changes the phase of all preceding consonant gestures with respect to the vowel. For a syllable coda position (VCC), both VC and CC coordination involve anti-phase target states. Hence, there is no competition between different coupling states and no c-center effect (for a more advanced overview of this modeling approach see Saltzman et al., 2006) as predicted by Browman and Goldstein.

A different model of coarticulation proposed by Öhman (1967) postulated two different modes for vowels and consonants. A vowel1-consonant-vowel2 (V1CV2) sequence is assumed to emerge from a vowel cycle, i.e. from the transition between the two vowel targets with a superimposed consonant cycle. Measuring the coarticulatory variability in V1CV2-sequences in Spanish and Catalan, Recasens $(1987,2002)$ observed that the influence of V2 on V1 and on C was strongly dependent on the consonant C. He interpreted this phenomenon as the result of consonant specific constraints imposing more or less resistance against the variability (called DAC, DAC, Degree of Articulatory Constraint) induced by coarticulation.

The integration of ideas of motor planning into theories of speech motor control started only in the late eighties. Whalen (1990) suggested that coarticulation may be "largely" planned. The concept of optimality was invoked by Keating (1988) in her window model in which sounds have a certain target window in the articulatory or acoustic domain. Planning a speech sequence consists of finding the optimal path through all the intervals

Similarly, the phonological inventory of a language (i.e. the number of elementary sounds and their proximity) may be a constraint on coarticulation (Manuel \& Krakow, 1984; Manuel, 1990). A language with a small number of well distinct phonemes may allow for more variability and stronger coarticulation than a language with a more crowded 
vowel space. The structure of the language may also constrain the length of the units that are planned within a sequence (Ma et al., in press).

\section{Sequencing and Coarticulation in Limb Movements}

In contrast to the generation of phonemes in speech, the execution of sequences of object oriented limb movements is usually assumed to be less time critical. Coarticulation may, however, be relevant when temporally contiguous movements are made. Consider two different movements in our example of waiter and patron. If the waiter reaches for an inverted glass to pour water for the patron, he will approach the glass with his hand in a thumb-down position in order to hold it in thumb-up position when pouring water into it. In contrast, if the waiter just moves the same glass from one end of the counter to another, he will grasp it in thumb-up hand position. Obviously, the choice of the first motor act is adapted with respect to the goal of the second movement, enabling a comfortable end state (Rosenbaum et al., 2006).

Similar to those daily life observations, experiments with various motion tasks showed that movement parameters like hand shaping, peak aperture, and reaching duration of an initial reaching and grasping submovement are affected in various ways by the movement that follows (Ansuini et al., 2006, 2008; Armbrüster \& Spijkers, 2006). Throwing or placing an object under high or low accuracy constraints, for instance, affects hand shaping during the approach phase. Specifically, the hand posture for grasping the object is attained early during the reach and remains almost unchanged during the reach when the end-goal does not require accurate manipulation. Furthermore, the speed of the reaching movement is also affected by the precision requirements of the subsequent movement. Low accuracy leads to fast, high accuracy to slow reaching movements. The energetic efficiency of coarticulation in limb movements was addressed by Klein, Breteler et al. (2003) who found that anticipatory modifications of the terminal arm posture of the first sub-movement in a 3D drawing sequence maximized end state comfort and energetic efficiency of the overall movement.

The reviewed evidence for coarticulation comes from temporally discrete, object- 
oriented limb movements, which are not subject to rhythmic timing constraints. More similar to speech, perhaps, are the rhythmic movement sequences involved in making music. Playing the piano requires bimanual key presses within a strict temporal schema in order to produce enjoyable music. In a review of this task that focused on coarticulation of fingers, Engel (1997) found anticipatory movements that subserved the temporal or spatial requirements of the musical task. To adhere to the temporal constraints of the piano piece, players had to perform a so-called intermediary "thumb-under maneuver" with hand repositioning for specific sequences of notes. In other cases, with the same starting sequence, this reconfiguration was not necessary. This shows that, as a matter of principle, the hand motor system has the capacity of executing sequential elements in an overlapping fashion.

Another scenario of limb movements with a strong linguistic component is the production of sign language, which is easily accessible to observation. Jerde et al. (2003a) evaluated the impact of coarticulation in finger spelling by measuring movements of fingers and wrist. They found forward and reverse influences across finger-generated letters analogous to the patterns found in speech. In addition to the tendency to reduce differences between sequential finger shapes - called assimilation by the authors - they identified a second class of coarticulation events which result in an accentuation of differences between shapes. This so-called dissimilation may support visual discrimination of words and recognition by the observer. Consequently, dissimilation was predominant for the index and middle finger joints which are known to be fundamental for letter recognition (Jerde et al., 2003b).

The reviewed aspects of coarticulation in limb motor control appear to be primarily reflective of efficiency and end state comfort and generally less time critical than related effects in speech production. It is quite possible, however, that closer analogies to speech production may be discovered if coarticulation were studied in more complex manipulation tasks that require fluent, coordinated action. This may be an interesting field of research in which the domain of limb motor control may derive inspiration from work on speech production. 


\section{Control and Biomechanics}

We return to our glass-of-water example for one last time. At some point, the physical movement of the arm must be brought about. To accelerate and then decelerate the arm, the actor must overcome the inertia of the arm. Because the arm is a multi-link kinematic chain, the different joints are mechanically coupled. Torque actively generated at any individual joint leads to reactive torques at the other joints. Gravitational forces are at work all the time, of course, but their impact on the arm's degrees of freedom varies as the joint configuration changes. When the actor has grasped the glass, the force relationships change again, as the glass adds inertial and gravitational load to the arm.

Moreover, to keep a firm grip of the glass, the frictional force exercised by the hand onto the glass must be sufficient to transmit the acceleration and deceleration of the hand onto the glass. Moving a glass filled with water also entails solving complex problems of coupled rigid body and fluid dynamics. Mechanical problems of this general nature must be solved similarly by the speech articulatory system. Although the speech apparatus does not typically deal with unknown external objects, problems of inertia are important given the high movement rates of the articulators. Moreover, the speech articulatory system has some particularities such as the deformable soft-tissue structures of lips and tongue.

Gravity is a basic mechanical constraint for movements. How gravity matters is reflected in the modification of movement kinematics when the orientation of a movement changes relative to the gravity vector (Papaxanthis et al., 1998; Pozzo et al., 1998). Similar observations have been made for arm movements in a pointing task by Smetanin and Popov (1997) and for jaw movements during speech production by Shiller et al. (1999). These authors compared the terminal positions reached at the end of the movement, when participants' bodies were oriented either vertically or horizontally in the gravitational field. In both movement domains the terminal positions differed consistently with respect to the effect of gravity: arm movement amplitude was smaller and jaw position lower, when movements were made in opposition to the gravity vector. However, these differences remained within an acceptable range and the movement goals were attained under all conditions. In the case of speech, intelligibility was preserved independently of the head's 
orientation. Stone et al. (2007) observed similar differences in tongue movements during speech production in upright versus supine position. Simulations based on a realistic 3D biomechanical tongue model that employed the same motor commands for upright and supine position reproduced these differences (Buchaillard et al., 2009). All these observations suggest that for both limb and speech movements, the motor system does not adjust motor commands to create movements that are invariant against changes in orientation relative to gravity, but endows movement with a sufficient amount of stability to remain functional under such varied conditions.

Other than the influence of gravity, the mechanical constraints relevant to arm and limb movements are quite different from those relevant to speech movements. Arm and limb movements face considerable changes in inertia and interaction torques across workspace. An indirect indication of this fact emerges from the perturbation experiments of Shadmehr and Mussa-Ivaldi (1994) discussed earlier. After learning to adapt to an external force field, which was then removed, participants displayed after-effects both in the right part of the work space, which they had explored during adaptation as well as in the left part of the work space, which they had not. The after-effects differed in the two hemi-spaces in a way that was consistent with differences in joint geometry. Hence, the movement accuracy obtained under normal conditions across the entire work space must be achieved in a way that takes into account these mechanical differences.

Arm movements may be oriented toward physical objects, another distinction from speech movements. Johansson and Westling (1984) showed that participants who move their hand while holding slippery objects between their fingers adjust the grip force to the requirements of friction and object inertia. Similarly, Zatsiorsky et al. (2005) established a phase synchronized modulation of the grip force applied to a vertically oriented handle that participants moved cyclically up and down.

Speech articulators are, of course, also affected by gravity and inertia. Humans are able to speak while running or jumping or being accelerated in a carousel. Hence, the speech motor system can be stabilized in order to achieve the goals of the task under variable conditions. There are not many quantitative studies of the underlying control 
strategies. One contribution was made by Shiller et al. (2001) who studied the effects of head acceleration on jaw movements during speech production. Their results are consistent with the idea that motor commands are adjusted to reduce the effect of acceleration.

The specificity of speech production arises from the intrinsic biomechanical properties of the speech articulators. First, the typical high rate of speech leads to large accelerations. The tongue is a hydrostat, i.e. a soft body with highly complex, typically non linear, physical properties and deformation capabilities (Gerard et al., 2005). This articulator is supported by the mandible, a rigid body with dynamical properties that differ strongly from the ones of the tongue. Hence, the mechanical coupling between these two articulators cannot be described by conventional rigid-body mechanical laws. Another characteristic of tongue and lip movements is that these articulators are most of the time in contact with other parts of the vocal tract: the teeth, the palate, the velum, and the pharyngeal walls for the tongue, and the lower lip with the upper lip or the teeth. Hence, the mechanical boundary conditions and the effective number of degrees of freedom vary over the course of a movement. Finally, the speech articulators are also under the influence of pressure forces that arise from the propagation of airflow that is responsible for the generation of acoustic waves in the vocal tract.

The impact of these specific biomechanical properties of speech articulators has recently been studied. This has made it possible to develop realistic, complex, biomechanical models of the articulators (Wilhelms-Tricarico, 1995; Payan \& Perrier, 1997; Dang \& Honda, 2004; Gerard et al., 2006; Buchaillard et al., 2009). It has been shown, for instance, that trajectories of certain sounds are largely influenced by muscle anatomy and tongue-palate interactions (Perrier et al., 2003), as well as by fluid-soft tissues interactions (Perrier et al., 2000), and that velocity profiles can be determined by muscle fibers orientations (Payan \& Perrier, 1997). Perrier and Fuchs (2008) have suggested that speed-curvature relations may result from the intrinsic physical properties of the articulators. In the present volume, Nazari et al. examine the impact of the stress stiffening effect in contracted muscle tissues onto the achievement of lip protrusion and rounding. 


\section{Conclusion}

Looking at the full complexity of object-oriented limb movements as well as speech movements with an eye for analogies and contrasts, we found considerable convergence. Motor goals in both domains are clearly task-specific, but realized by coupling structures at the level of the effectors. Although target states organize goal-directed movement, specific control processes tune movement trajectories. The serial order of task states and their sequential production are central to speech and relevant to object-oriented action as well. Coordination among effectors has been studied in the two domains with strongly overlapping theoretical concepts and shared experimental methods.

Points of contrast also emerged from our comparative analysis. Inter-gestural coordination has not been studied much for object-oriented action, but is a central concern of researchers who try to understand how the elements of language are enacted during speech production. The fast, but task-dependent compensatory reactions to perturbations observed in speech production push the envelope of what is known from limb movements. Biomechanical constraints also differ in the two domains. In manipulatory movements, the effects of adaptation to external loads are a major concern. In speech production, the properties of soft tissue articulators and the sheer speed of the articulatory movements pose unique problems. A common thread of these points of divergence between the two domains may be the high rate of movement in speech production. Motor goals follow each other in a fast sequence of articulatory events. This may require more planning ahead, and more coordination among subsequent elements of the sequence allowing for fast anticipatory adjustments to predictable articulatory challenges.

Interchange between the research communities in these two domains of motor control has been a source of innovation in the past and will continue to be so in the future. For instance, the concept of stability emerged from studies of bimanual coordination but has led to profound inquiries into how the articulators are bound into stable patterns for brief time intervals before reconfiguring for the next gesture. Co-articulation may yet to become a source for a deeper understanding of the behavioral organization of object-oriented motor action. Moving between the two domains also provides useful perspectives in relation to 
the theoretical debates that we touched upon. In most cases, the best strategy has been to avoid simple yes/no dichotomies and instead uncover the underlying processes. The fluent movement from gesture to gesture that characterizes speech, for instance, suggests that movement is fundamentally continuous in nature, so that the traditional dichotomy of discrete and rhythmic movement may be about two particular limit cases that are not in fundamental opposition to each other. In both limb and speech motor control, the increasing availability of detailed process models leads to a new quality of theoretical debates by enabling researchers to explicitly formalize, quantify, and test different hypotheses. We believe that the confluence of convergent theoretical, conceptual, and empirical lines of thinking will greatly advance our understanding of the nature of voluntary movement across the two fields.

We have seen in many cases how the concepts that enable us to understand the motor control of speech production need to be tightly linked to the conceptual framework in which language itself is understood. The analogous issue in limb movement is, ultimately, the theoretical stance of embodied cognition which emphasizes that cognition manifests itself in its physical enaction in structured environments. From this point of view, cognitive processes are tightly linked to the sensory and motor surfaces and constrained by the organizational principles of nervous systems. Conversely, the embodiment stance implies that understanding the motor control of object-oriented actions requires a framework for how meaningful goal-oriented behavior emerges in structured environments from embodied cognitive systems. The shared theoretical language may be the language of timecontinuous neural dynamic processes that may capture the links across components and levels that enable fluid, goal-oriented behavior (Schneegans \& Schöner, 2008). If this analogy holds, it can also play back into the domain of language, in which it is becoming increasingly clear that the production of language is constrained by its embedding in situated communicative processes (Fowler et al., 2008). 


\section{References}

Abend, W., Bizzi, E., \& Morasso, P. (1982). Human arm trajectory formation. Brain, 105 (2), 331-348.

Amari, S. (1977). Dynamics of pattern formation in lateral-inhibition type neural fields. Biol Cybern, 27 (2), 77-87.

Ansuini, C., Giosa, L., Turella, L., Altoè, G., Castiello, U. (2008). An object for an action, the same object for other actions: effects on hand shaping. Exp Brain Res, 185 (1), $111-119$.

Ansuini, C., Santello, M., Massaccesi, S., Castiello, U. (2006). Effects of end-goal on hand shaping. J Neurophysiol, 95 (4), 2456-2465.

Armbrüster, C., \& Spijkers, W. (2006). Movement planning in prehension: do intended actions influence the initial reach and grasp movement? Motor Control, 10 (4), $311-329$.

Aronson, D. G., Doedel, E. J., \& Othmer, H. G. (1987). An analytical and numerical study of the bifurcations in a system with linearly coupled oscillators. Physica D, $25,20-104$.

Baer, T., Alfonso, P. J. \& Honda, K. (1988), Electromyography of the tongue muscles during vowels in /əpVp/ environment, RILP Tokyo 22, 7-19.

Bernstein, N. (1935). The problem of the interrelation of coordination and localization. Arch Biol Sci, 38, 15-59.

Bizzi, E., Accornero, N., Chapple, W., \& Hogan, N. (1984). Posture control and trajectory formation during arm movement. J Neurosci, 4 (11), 2738-2744.

Bladon, R.A.W. \& Fant, G. (1978). A two-formant model and the cardinal vowels. STLQPSR, 1 1-8.

Blumstein, S. E., \& Stevens, K. N. (1979). Acoustic invariance in speech production: evidence from measurements of the spectral characteristics of stop consonants. J Acoust Soc Am, 66 (4), 1001-1017.

Boë, L.-J., Perrier, P., \& Bailly, G. (1992). The geometric vocal tract variables controlled for vowel production: Proposals for constraining acoustic-to-articulatory inversion. J 
Phon, 20, 27-38.

Browman, C. P., \& Goldstein, L. M. (1986). Towards an Articulatory Phonology. Phonology Yearbook, 3, 219-252.

Browman, C. P., \& Goldstein, L. M. (2000). Competing constraints on intergestural coordination and self-organization of phonological structures. Bulletin de la Communication Parlée, 5, 25-34.

Buchaillard, S., Perrier, P., \& Payan, Y. (2009). A biomechanical model of cardinal vowel production: muscle activations and the impact of gravity on tongue positioning. $\mathbf{J}$ Acoust Soc Am, 126(4), 2033-2051.

Borden, G. J., Harris, K. S., \& Oliver, W. (1973). Oral feedback I. Variability of the effect of nerve-block anesthesia upon speech. J Phon, 1, 289-295.

Cai, S., Boucek, M., Ghosh, S. S., Guenther, F. H., \& Perkell, J. S. (2008). A system for online dynamic perturbation of formant trajectories \& results from perturbations of the Mandarin triphthong /iau/. In Proceedings of the 8th ISSP (pp. 65-68). Strasbourg, France.

Chomsky, N., \& Halle, M. (1968). The Sound Pattern of English. Harper \& Row: New York.

Cruse, H., Brüwer, M., \& Dean, J. (1993). Control of three-and four-joint arm movement: Strategies for a manipulator with redundant degrees of freedom. J Mot Behav, 25(3), 131-139.

Cummins, F., \& Port, R. F. (1998). Rhythmic constraints on stress timing in English. J Phon, 26 (2), 145-171.

Cutler, A., Eisner, F., McQueen, J. M., \& Norris, D. (in press). How abstract phonemic categories are necessary for coping with speaker-related variation. In C. Fougeron,

B. Kühnert, M. D’Imperio \& N. Vallée (Eds.) Papers in Laboratory Phonology 10. Mouton de Gruyter.

Dang, J., \& Honda, K. (2004). Construction and control of a physiological articulatory model. J Acoust Soc Am, 115 (2), 853-870.

Delattre, P., Liberman, A. M., \& Cooper, F. (1955). Acoustic loci and transitional cues 
for consonants. J Acoust Soc Am, 27, 769-773.

Desmurget, M., Pélisson, D., Rossetti, Y., \& Prablanc, C. (1998). From eye to hand: planning goal-directed movements. Neurosci Biobehav Rev, 22 (6), 761-788.

Engel, K. C., Flanders, M., Soechting, J. F. (1997). Anticipatory and sequential motor control in piano playing. Exp Brain Res, 113 (2), 189-199.

Fadiga, L., Fogassi, L., Pavesi, G., \& Rizzolatti, G. (1995). Motor facilitation during action observation: a magnetic stimulation study. J Neurophysiol, 73 (6), 2608-2611.

Fant, G. (1960). Acoustic Theory of Speech Production. Mouton: The Hague.

Feldman, A. G. (1986). Once more on the equilibrium-point hypothesis (lambda model) for motor control. Journal of Motor Behaviour, 18(1), 17-54.

Fitts, P. M. (1954). The information capacity of the human motor system in controlling the amplitude of movement. J Exp Psychol, 47 (6), 381-391.

Flanagan, J. R., \& Ostry, D. J. (1990). Experimental Robotics. In V. Hayward \& O. Khatib (Eds.), (pp. 594-613) Springer: London.

Flash, T., \& Hogan, N. (1985). The coordination of arm movements: an experimentally confirmed mathematical model. J Neurosci, 5 (7), 1688-1703.

Fowler, C. A. (1980). Coarticulation and theories of extrinsic timing control. J Phon, 8, $113-133$.

Fowler, C. A. (1986). An event approach to the study of speech perception from a directrealist perspective. J Phon, 14, 3-28.

Fowler, C. A. (1991). Auditory perception is not special: we see the world, we feel the world, we hear the world. J Acoust Soc Am, 89 (6), 2910-2915.

Fowler, C. A., Richardson, M. J., Marsh, K. L., \& Shockley, K. (2008). Language use, coordination, and the emergence of cooperative action. In A. Fuchs \& V. Jirsa (Eds.), Coordination: Neural, Behavioral and Social Dynamics. (pp. 261-279). Berlin: Springer Verlag.

Franz, E., Zelaznik, H., \& Smith, A. (1992). Evidence of common timing processes in the control of manual, orofacial, and speech movements. J Mot Behav, 24 (3), 281-287.

Fuchs, S. (2005). Articulatory correlates of the voicing contrast in alveolar obstruent 
production in German. ZAS papers in Linguistics 41 .

Fujimura, O. (1986). Relative invariance of articulatory movements. In Perkell J.S \& Klatt D.H (Eds), Invariance and Variability in Speech Processes (pp. 226-242). Hillsdale, N.J.: Lawrence Erlbaum.

Galantucci B., Fowler, C.A. \& Turvey, M.T. (2006). The motor theory of speech perception reviewed. Psychonomic Bulletin \& Review 13 (3), 361-377.

Gay, T., Lindblom, B., \& Lubker, J. (1981). Production of bite-block vowels: Acoustic equivalence by selective compensation, J Acoust Soc Am 69, 802-810.

Gerard, J.-M., Ohayon, J., Luboz, V., Perrier, P., \& Payan, Y. (2005). Non-linear elastic properties of the lingual and facial tissues assessed by indentation technique. Application to the biomechanics of speech production. Med Eng Phys, 27 (10), 884892.

Gerard, J.-M., Perrier, P., \& Payan, Y. (2006). 3D biomechanical tongue modelling to study speech production. In J. Harrington \& M. Tabain (Eds.), Speech Production: Models, Phonetic Processes, and Techniques (pp. 85-102). New-York, USA: Psychology Press.

Gibson, J. J. (1966). The Senses Considered as Perceptual Systems. Houghton Mifflin: Boston.

Gomi, H., \& Kawato. (1996). Equilibrium-point control hypothesis examined by measured arm stiffness during multijoint movement. Science, 272 (5258), 117-120.

Gracco, V. L. (1988). Timing factors in the coordination of speech movements. J Neurosci, 8 (12), 4628-4639.

Gribble, P. L., \& Ostry, D. J. (1996). Origins of the power law relation between movement velocity and curvature: modeling the effects of muscle mechanics and limb dynamics. J Neurophysiol, 76 (5), 2853-2860.

Gribble, P. L., Ostry, D. J., Sanguineti, V., \& Laboissière, R. (1998). Are complex control signals required for human arm movement? J Neurophysiol, 79 (3), 1409-1424.

Grossberg, S., Pribe, C., \& Cohen, M. A. (1997). Neural control of interlimb oscillations. I. Human bimanual coordination. Biol Cybern, 77 (2), 131-140. 
Guenther, F. H. (1995). Speech sound acquisition, coarticulation, and rate effects in a neural network model of speech production. Psychol Rev, 102 (3), 594-621.

Guenther, F. H., Hampson, M., \& Johnson, D. (1998). A theoretical investigation of reference frames for the planning of speech movements. Psychol Rev, 105 (4), $611-633$.

Harris, C. M., \& Wolpert, D. M. (1998). Signal-dependent noise determines motor planning. Nature, 394 (6695), 780-784.

Helmuth, L. L., \& Ivry, R. B. (1996). When two hands are better than one: reduced timing variability during bimanual movements. J Exp Psychol Hum Percept Perform, 22 (2), 278-293.

Henke, W. (1966). Dynamic Articulatory Model of Speech Production Using Computer Simulation. Unpublished doctoral dissertation, MIT.

Hermens, F., \& Gielen, S. (2004). Posture-based or trajectory-based movement planning: a comparison of direct and indirect pointing movements. Exp Brain Res, 159 (3), 340348.

Hogan, N. (1984). An organizing principle for a class of voluntary movements. J Neurosci, 4 (11), 2745-2754.

Hogan, N., \& Sternad, D. (2007). On rhythmic and discrete movements: reflections, definitions and implications for motor control. Exp Brain Res, 181 (1), 13-30.

Houde, J. F., \& Jordan, M. I. _(1998). Sensorimotor adaptation in speech production, Science; $279,1213-1216$.

Hsu, W.-L., Scholz, J. P., Schöner, G., Jeka, J. J., \& Kiemel, T. (2007). Control and estimation of posture during quiet stance depends on multijoint coordination. $\mathbf{J}$ Neurophysiol, 97 (4), 3024-3035.

Hughlings Jackson, J. (1889). On the comparative study of disease of the nervous system. British Medical Journal, 355-362, Aug. 17, 1889.

Iacoboni, M., Woods, R. P., Brass, M., Bekkering, H., Mazziotta, J. C., \& Rizzolatti, G. (1999). Cortical mechanisms of human imitation. Science, 286 (5449), 2526-2528. Isei-Jaakkola, T. (2004). Lexical Quantity in Japanese and Finnish. Unpublished doctoral 
dissertation, University of Helsinki, Faculty of Behavioural Sciences, Department of Phonetics.

Ivry, R. B. (1996). The representation of temporal information in perception and motor control. Curr Opin Neurobiol, 6 (6), 851-857.

Ivry, R. B., Diedrichsen, J., Spencer, R., Hazeltine, E., \& Semjen, A. (2004). A cognitive neuroscience perspective on bimanual coordination and interference. In S. P. Swinnen \& J. Duysens (Eds.), Neuro-Behavioral Determinants of Interlimb Coordination - An Interdisciplinary Approach (pp. 259-295). Dordrecht, The Netherlands: Kluwer Academic Publishers.

Ivry, R. B., \& Hazeltine, R. E. (1995). Perception and production of temporal intervals across a range of durations: evidence for a common timing mechanism. $\mathrm{J}$ Exp Psychol Hum Percept Perform, 21 (1), 3-18.

Ivry, R. B., \& Richardson, T. C. (2002). Temporal control and coordination: the multiple timer model. Brain Cogn, 48 (1), 117-132.

Ivry, R. B., \& Spencer, R. M. C. (2004). The neural representation of time. Curr Opin Neurobiol, 14 (2), 225-232.

Jeannerod, M. (1984). The timing of natural prehension movements. J Mot Behav, 16 (3), $235-254$.

Jerde, T. E., Soechting, J. F., Flanders, M. (2003a). Coarticulation in fluent fingerspelling. J Neurosci, 23 (6), 2383-2393.

Jerde T. E., Soechting, J. F., Flanders, M. (2003b). Biological constraints simplify the recognition of hand shapes. IEEE Trans Biomed Eng, 50 (2), 265-269.

Johansson, R. S., \& Westling, G. (1984). Roles of glabrous skin receptors and sensorimotor memory in automatic control of precision grip when lifting rougher or more slippery objects. Exp Brain Res, 56 (3), 550-564.

Kawato, M., Maeda, Y., Uno, Y., \& Suzuki, R. (1990). Trajectory formation of arm movement by cascade neural network model based on minimum torque-change criterion. Biol Cybern, 62, 275-288.

Keating, P. (1988). The window model of coarticulation: articulatory evidence. CLA 
Working Papers in Phonetics, 69, 3-29.

Kelso, J. A. (1984). Phase transitions and critical behavior in human bimanual coordination. Am J Physiol, 246 (6 Pt 2), R1000-R1004.

Kelso, J. A., Saltzman, E. L., \& Tuller, B. (1986). The dynamical perspective on speech production: data and theory. J Phon, 14, 29-59.

Kelso, J. A., Scholz, J. P., \& Schöner, G. (1986). Nonequilibrium phase transitions in coordinated biological motion: critical fluctuations. Physical Letters A, 118 (6), 279-284.

Kelso, J. A., Scholz, J. P., \& Schöner, G. (1988). Dynamics governs switches among patterns of coordination in biological movement. Physical Letters A, 134, 8-12.

Kelso, J. A., Southard, D. L., \& Goodman, D. (1979). On the nature of human interlimb coordination. Science, 203 (4384), 1029-1031.

Kelso, J.A.S. \& Tuller, B. (1983). Compensatory articulation under conditions of reduced afferent information: A dynamic formulation. JSLHR, 26, 217-224.

Kelso, J. A., Tuller, B., Vatikiotis-Bateson, E., \& Fowler, C. A. (1984). Functionally specific articulatory cooperation following jaw perturbations during speech: evidence for coordinative structures. J Exp Psychol Hum Percept Perform, 10 (6), 812-832.

Klein Breteler, M. D., Hondzinski, J. M., Flanders, M. (2003). Drawing sequences of segments in 3D: kinetic influences on arm configuration. J Neurophysiol, 89 (6), $3253-3263$

Krishnamoorthy, V., Yang, J.-F., \& Scholz, J. P. (2005). Joint coordination during quiet stance: effects of vision. Exp Brain Res, 164 (1), 1-17.

Kuhl, P.K. (1991). Human adults and human infants show a perceptual magnet effect for the prototypes of speech categories, monkeys do not. Perception and Psychophysics, $50,93-107$

Laboissière, R., Ostry, D. J., \& Feldman, A. G. (1996). The control of multi-muscle systems: human jaw and hyoid movements. Biol Cybern, 74 (4), 373-384.

Ladefoged, P. (2000). Vowels and Consonants: An Introduction to the Sounds of Languages. Oxford: Blackwell Publishing. 
Large, E. W., \& Palmer, C. (2002). Perceiving temporal regularity in music. Cognitive Science, 26, 1-37.

Latash, M. L., Scholz, J. P., \& Schöner, G. (2007). Toward a new theory of motor synergies. Motor Control, 11 (3), 276-308.

Liberman, A. M., Cooper, F. S., Shankweiler, D. P., \& Studdert-Kennedy, M. (1967). Perception of the speech code. Psychol Rev, 74 (6), 431-461.

Liberman, A. M., \& Mattingly, I. G. (1985). The motor theory of speech perception revised. Cognition, 21 (1), 1-36.

Lindblom, B. (1988). Phonetic invariance and the adaptive nature of speech. In Elsendoorn \& Bouma (Eds.), Working Models of Human Perception. (p. 139-173). Academic Press: London.

Lindblom, B. (1990). Explaining phonetic variation: a sketch of the H\&H theory. In W. Hardcastle \& A. Marchal (Eds.), Speech Production and Speech Modeling. (p. 403-439). Kluwer: Dordrecht.

Lotto, A., Hickok, G., \& Holt, L. (2009). Reflections on mirror neurons and speech perception. Trends in Cognitive Sciences, 13, 10-114.

Lund, J.P., \& Kolta, A. (2006). Brainstem circuits that control mastication: Do they have anything to say during speech. Journalf of Communication Disorders, 39(5), 381390.

MacNeilage, P. F. (1970). Motor control of serial ordering of speech. Psychological Review, 77, 182-196.

MacNeilage, P.F (1998). The Frame/Content theory of evolution of speech production. Behavioral and Brain Sciences, 21, 499-546.

MacNeilage, P. F., \& Davis, B. L. (2000). On the origin of internal structure of word forms. Science, 288 (5465), 527-531.

Maeda, S. \& Honda, K. (1994). From EMG to formants patterns of vowels:the implication of vowel spaces. Phonetica, 51(1-3), 17-29.

Manuel, S. (1990). The role of contrast in limiting vowel-to-vowel coarticulation in different languages. J Acoust Soc Am, 88, 1286-1298. 
Manuel, S., \& Krakow, R. (1984). Universal and language particular aspects of vowelto-vowel coarticulation. Haskins Laboratories Status Reports on Speech Research, SR-77/78, 69-78.

Marin, S., \& Pouplier, M. (2010). Temporal organization of complex onsets and codas in American English: Testing the predictions of a gestural coupling model. Motor Control 14(3), 380-407.

Martin, V., Scholz, J. P., \& Schöner, G. (2009). Redundancy, self-motion, and motor control. Neural Comput, 21 (5), 1371-1414.

McFarland, D., Baum, S. \& Chabot, C. (1996). Speech compensation to structural modifications of the oral cavity. Journal of the Acoustical Society of America, 100, 1093-1104.

McGurk, H., \& MacDonald, J. (1976). Hearing lips and seeing voices. Nature, 264 (5588), 746-748.

Mechsner, F., Kerzel, D., Knoblich, G., \& Prinz, W. (2001). Perceptual basis of bimanual coordination. Nature, 414 (6859), 69-73. Morasso, P. (1981). Spatial control of arm movements. Exp Brain Res, 42 (2), 223-227.

Munhall, K. G., \& Kelso, J. A. (1985). The role of similarity analysis in understanding movement. J Mot Behav, 17 (4), 493-498.

Munhall, K. G., Löfqvist, A., \& Kelso, J. A. (1994). Lip-larynx coordination in speech: effects of mechanical perturbations to the lower lip. J Acoust Soc Am, 95 (6), $3605-3616$

Nam, H., \& Saltzman, E. L. (2003). A competitive, coupled oscillator model of syllable structure. In Proceedings of the 15th International Congress of Phonetic Sciences. Barcelona (p. 2253-2256).

Nazari, M. A., Perrier, P., Chabanas, M., \& Payan, Y. (this volume). Shaping by stiffening: a modeling study for lips.

Ohala, J. J. (1983). The phonological end justifies any means . In S. Hattori \& K. Inoue (Eds.), Proc. of the XIIIth Int. Cong. of Linguists (pp. 232-243). Tokyo.

Ohman, S. E. (1967). Studies of articulatory coordination. STL-QPSR 8(1), 15-20. 
Papaxanthis, C., Pozzo, T., Vinter, A., \& Grishin, A. (1998). The representation of gravitational force during drawing movements of the arm. Exp Brain Res, 120 (2), $233-242$.

Paulignan, Y., MacKenzie, C., Marteniuk, R., \& Jeannerod, M. (1991). Selective perturbation of visual input during prehension movements. 1. The effects of changing object position. Exp Brain Res, 83 (3), 502-512.

Payan, Y., \& Perrier, P. (1997). Synthesis of V-V sequences with a 2D biomechanical tongue model controlled by the equilibrium point hypothesis. Speech Comm, 22 (2-3), 185-205.

Perkell, J. S., \& Klatt, D. (Eds.). (1986). Invariance and Variability of Speech Processes. Lawrence Erlbaum Association.

Perrier, P. (2005). Control and representations in speech production. Special Issue on Speech production and perception: Experimental analyses and models (S. Fuchs, P. Perrier \& B. Pompino-Marschall, eds.), .ZAS Papers in Linguistics.40, 190-132.

Perrier, P. (2006). About speech motor control complexity. In J. Harrington \& M. Tabain (Eds.), Speech Production: Models, Phonetic Processes, and Techniques (pp. 13-25). Psychology Press: Sydney.

Perrier, P., \& Fuchs, S. (2008). Speed-curvature relations in speech production challenge the 1/3 power law. J Neurophysiol, 100 (3), 1171-1183.

Perrier, P., \& Ma, L. (2008). Speech planning for V1CV2 sequences: Influence of the planned sequence. In Proceedings of the 8th ISSP (pp. 69-72). Strasbourg, France.

Perrier, P., Payan, Y., Perkell, J. S., Zandipour, M., Pelorson, X., Coisy, V. \& Matthies, M. (2000). An attempt to simulate fluid-walls interactions during velar stops. In Proceedings of the 5th ISSP. Munich, Germany.

Perrier, P., Payan, Y., Zandipour, M., \& Perkell, J. (2003). Influences of tongue biomechanics on speech movements during the production of velar stop consonants: a modeling study. J Acoust Soc Am, 114 (3), 1582-1599.

Petrides, M., Cadoret, G. \& Mackey, S. (2005). Orofacial somatomotor responses in the macaque monkey homologue of Broca's area. Nature, 435, 1235-1238. 
Polit, A., \& Bizzi, E. (1979). Characteristics of motor programs underlying arm movements in monkeys. J Neurophysiol, 42 (1 Pt 1), 183-194.

Pozzo, T., Papaxanthis, C., Stapley, P., \& Berthoz, A. (1998). The sensorimotor and cognitive integration of gravity. Brain Res Brain Res Rev, 28 (1-2), 92-101.

Purcell, D.W, \& Munhall, K.G. (2006). Compensation following real-time manipulation of formants in isolated vowels. J Acoust Soc Am, 119(4), 2288-2297.

Recasens, D. (1987). An acoustic analysis of V-to-C and V-to-V coarticulatory effects in Catalan and Spanish VCV sequences. J Phon, 15, 299-312.

Recasens, D. (2002). An EMA study of VCV coarticulatory direction. J Acoust Soc Am, $111(6), 2828-2841$.

Rizzolatti, G., Fogassi, L., \& Gallese, V. (2001). Neurophysiological mechanisms underlying the understanding and imitation of action. Nat Rev Neurosci, 2 (9), $661-670$.

Rochet-Capellan, A., \& Schwartz, J.-L. (2007). An articulatory basis for the labialto-coronal effect: /pata/ seems a more stable articulatory pattern than /tapa/. J Acoust Soc Am, 121 (6), 3740-3754.

Rosenbaum, D. A., Cohen, R. G., Meulenbroek, R. G., and Vaughan, J. (2006). Plans for grasping objects. In A. Feldman (Ed.), Progress in Motor Control. Springer.

Saltzman, E. L. (1986). Task dynamic coordination of the speech articulators: A preliminary model. Exp Brain Res, 15, 129-144.

Saltzman, E. L., Kubo, M., Tsao, C.-C. (2006) Controlled variables, the uncontrolled manifold, and the task-dynamics model of speech production. In: P. Divenyi, S., Greenberg \& G. Meyer (Eds.) Dynamics of speech production and perception. (pp. 21-31) IOP Press: Amsterdam.

Saltzman, E. L., Löfqvist, A., Kinsella-Shaw, J., Rubin, P. E., \& Kay, B. A. (1992). Perturbation study of lip-larynx coordination. In Proceedings of the ICSLP (p. 19-22).

Saltzman, E. L., \& Munhall, K. G. (1989). A dynamical approach to gestural patterning in speech production. Ecological Psychology, 1 (4), 333-382. 
Saltzman, E. L., Nam, H., Goldstein, L., \& Byrd, D. (2006). The distinctions between state, parameter, and graph dynamics in sensorimotor control and coordination. In M. Latash \& F. Lestienne (Eds.), Motor Control and Learning (pp. 61-73). Springer: New York.

Sato, M., Schwartz, J.-L., Abry, C., Cathiard, M., \& Loevenbruck, H. (2006). Multistable syllables as enacted percept: A source of an asymmetric bias in the verbal transformation effect. Perception \& Psychophysics, 68(3), 458-474.

Savariaux C., Perrier P. \& Orliaguet J.P. (1995) Compensation Strategies for a Lip-tube Perturbation of the Rounded Vowel [u]. J Acoust Soc Am, 98 (5), 2428-2442.

Schaal, S., \& Sternad, D. (2001). Origins and violations of the 2/3 power law in rhythmic three-dimensional arm movements. Exp Brain Res, 136, 60-72.

Schaal, S., Sternad, D., Osu, R., \& Kawato, M. (2004). Rhythmic arm movement is not discrete. Nat Neurosci, 7 (10), 1136-1143.

Schneegans, S., \& Schöner, G. (2008). Field theory as a framework for understanding embodied cognition. In P. Calvo \& T. Gomila (Eds.), Handbook of Cognitive Science: An Embodied Approach.

Scholz, J. P., \& Schöner, G. (1999). The uncontrolled manifold concept: identifying control variables for a functional task. Exp Brain Res, 126 (3), 289-306.

Scholz, J. P., Schöner, G., \& Latash, M. L. (2000). Identifying the control structure of multijoint coordination during pistol shooting. Exp Brain Res, 135 (3), 382-404.

Schöner, G. (1989). Learning and recall in a dynamic theory of coordination patterns. Biol Cybern, 62 (1), 39-54.

Schöner, G. (1994). Dynamic theory of action-perception patterns: The time-beforecontact paradigm. Human Movement Science, 3:415-439, 1994.

Schöner, G. (1995). Recent developments and problems in human movement science and their conceptual implications. Ecological Psychology, 7, 291-314.

Schöner, G. (2002). Timing, clocks, and dynamical systems. Brain Cogn, 48 (1), 31-51.

Schöner, G., Haken, H., \& Kelso, J. A. (1986). A stochastic theory of phase transitions in human hand movement. Biol Cybern, 53 (4), 247-257. 
Schöner, G., \& Kelso, J. A. (1988). Dynamic pattern generation in behavioral and neural systems. Science, 239 (4847), 1513-1520.

Schöner, G., Martin, V., Reimann, H., Scholz, J.P. : Motor equivalence and the uncontrolled manifold. In: R. Sock, S. Fuchs, Y. Laprie (Eds.): Proceedings of the International Seminar on Speech Production (ISSP 2008) in Strassbourg (pp. 23-28), INRIA.

Schwartz, J.J., Basirat, A., Ménard, L. \& Sato, M. (2010 in press). The Perception-forAction-Control Theory (PACT): a perceptuo-motor theory of speech perception. Journal of Neurolinguistics.

Shadmehr, R., \& Mussa-Ivaldi, F. A. (1994). Adaptive representation of dynamics during learning of a motor task. J Neurosci, 14 (5 Pt 2), 3208-3224.

Shiller, D. M., Ostry, D. J., \& Gribble, P. L. (1999). Effects of gravitational load on jaw movements in speech. J Neurosci, 19 (20), 9073-9080.

Shiller, D. M., Ostry, D. J., Gribble, P. L., \& Laboissière, R. (2001). Compensation for the effects of head acceleration on jaw movement in speech. J Neurosci, 21 (16), 64476456.

Smetanin, B. N., \& Popov, K. E. (1997). Effect of body orientation with respect to gravity on directional accuracy of human pointing movements. Eur J Neurosci, 9 (1), 7-11.

Soechting, J. F., Buneo, C. A., Herrmann, U., \& Flanders, M. (1995). Moving effortlessly in three dimensions: does Donders' law apply to arm movement? J Neurosci, 15 (9), 6271-6280.

Soechting, J. F., \& Lacquaniti, F. (1981). Invariant characteristics of a pointing movement in man. J Neurosci, 1 (7), 710-720.

Stetson, R. H. (1928). Motor Phonetics: a study of speech movements in action. Archives Néerlandaises de Phonétique Expérimentale, 3, 1-219.

Stevens, K. N. (1972). The quantal nature of speech: Evidence from articulatory-acoustic data. In J. E. David \& P. Denes (Eds.), Human Communication: A Unified View. (pp. 51-66). Mc Graw Hill: New York.

Stevens, K. N. (1989). On the quantal nature of speech. J Phon, 17, 3-45. 
Stevens, K. N., \& Blumstein, S. E. (1978). Invariant cues for place of articulation in stop consonants. J Acoust Soc Am, 64 (5), 1358-1368.

Stone, M., Stock, G., Bunin, K., Kumar, K., Epstein, M., Kambhamettu, C., et al. (2007). Comparison of speech production in upright and supine position. J Acoust Soc Am, $122(1), 532-541$.

Strange, W., Jenkins, J. J., \& Johnson, T. L. (1983). Dynamic specification of coarticulated vowels. J Acoust Soc Am, 74 (3), 695-705.

Sussman, H. M., Dalston, E., \& Gumbert, S. (1998). The effect of speaking style on a locus equation characterization of stop place of articulation. Phonetica, 55 (4), 204-225.

Todorov, E., \& Jordan, M. I. (2002). Optimal feedback control as a theory of motor coordination. Nat Neurosci, 5 (11), 1226-1235.

Tremblay, S., Shiller, D. M., \& Ostry, D. J. (2003). Somatosensory basis of speech production. Nature, 423 (6942), 866-869.

Tremblay S., Houle G., Ostry D.J. (2008). Specificity of speech motor learning. J Neurosci, 28, 2426-243.

Tseng, Y.-W., Scholz, J. P., Schöner, G., \& Hotchkiss, L. (2003). Effect of accuracy constraint on joint coordination during pointing movements. Exp Brain Res, 149 (3), 276-288.

Turvey, M. T. (1990). Coordination. Am Psychol, 45 (8), 938-953.

Uno, Y., Kawato, M., \& Suzuki, R. (1989). Formation and control of optimal trajectory in human multijoint arm movement -minimum torque-change model. Biol Cybern, 61, 89-101.

Vindras, P., Desmurget, M., Prablanc, C., \& Viviani, P. (1998). Pointing errors reflect biases in the perception of the initial hand position. J Neurophysiol, 79 (6), 32903294.

Viviani, P., \& Flash, T. (1995). Minimum-jerk, two-thirds power law, and isochrony: converging approaches to movement planning. J Exp Psychol Hum Percept Perform, 21 (1), 32-53. 
Viviani, P., \& Stucchi, N. (1992). Biological movements look uniform: evidence of motor-perceptual interactions. J Exp Psychol Hum Percept Perform, 18 (3), $603-623$.

Whalen, D. H. (1990). Coarticulation is largely planned. J Phon, 18, 3-35.

Wilhelms-Tricarico, R. (1995). Physiological modeling of speech production: methods for modeling soft-tissue articulators. J Acoust Soc Am, 97 (5 Pt 1), 3085-3098.

Wing, A. M., \& Kristofferson, A. B. (1973). Response delays and the timing of discrete motor responses. Perception \& Psychophysics, 14, 5-12.

Wolpert, D. M., Ghahramani, Z., \& Jordan, M. I. (1995). An internal model for sensorimotor integration. Science, 269 (5232), 1880-1882.

Yamanishi, J., Kawato, M., \& Suzuki, R. (1980). Two coupled oscillators as a model for the coordinated finger tapping by both hands. Biol Cybern, 37 (4), 219-225.

Zatsiorsky, V. M., Gao, F., \& Latash, M. L. (2005). Motor control goes beyond physics: differential effects of gravity and inertia on finger forces during manipulation of hand-held objects. Exp Brain Res, 162 (3), 300-308.

Ziegler, W. (2003). Speech motor control is task-specific: Evidence from dysarthria and apraxia of speech. Aphasiology, 17(1), p. 3-36 
Author Note

This work was supported by a grant from the French-German University

(Saarbrücken) to the PILIOS project and by the German Federal Ministry of Education and Research to the PB1 project at ZAS. The authors also acknowledge support from the German Federal Ministry of Education and Research within the National Network Computational Neuroscience-Bernstein Fokus: "Learning behavioral models: From human experiment to technical assistance", grant FKZ 01GQ0951.

FOOT NOTES 
${ }^{\mathrm{i}}$ An effector system is redundant when more degrees of freedom are available than needed to achieve a particular task (Cruse et al., 1993; Martin et al., 2009).

ii These important issues are not addressed in this paper. We have addressed them more specifically in other publications (Perrier, 2006) 